\title{
Erratum to: Migrants Suffering Violence While in Transit Through Mexico: Factors Associated with the Decision to Continue or Turn Back
}

Edson Servan-Mori • Rene Leyva-Flores • Cesar Infante Xibille · Pilar Torres-Pereda • Rodrigo Garcia-Cerde

Published online: 25 August 2013

(C) Springer Science+Business Media New York 2013

Erratum to: J Immigrant Minority Health

DOI 10.1007/s10903-012-9759-3

It has been noticed that there is a repetition in Table 2 headings of the original publication.

It should read as "Migrants who did stay in a migrant shelter $(\mathrm{n}=862)$ " on the second column, instead of "Migrants who did not stay in a migrant shelter ( $\mathrm{n}=$ 1,852)". The correct table is given below.

The online version of the original article can be found under doi:10. 1007/s10903-012-9759-3.

\section{E. Servan-Mori}

Centre for Evaluation and Survey Research, National Institute of Public Health, Cuernavaca, Mexico

R. Leyva-Flores $(\bowtie) \cdot$ C. I. Xibille $\cdot$ P. Torres-Pereda .

R. Garcia-Cerde

Centre for Health Systems Research, National Institute of Public

Health, Av. Universidad 655. Colonia Santa María Ahuacatitlán, Cuernavaca, Morelos, Mexico

e-mail: rene.leyva@insp.mx 
Table 2 Migrants sociodemographic characteristics, migratory experience, health and violence according to their stay (or not) in a migrant shelter

Migrants who did not stay in a migrant shelter
$(\mathrm{n}=1,852)$

Reported some kind of violence

\begin{tabular}{lll}
\hline No & Yes & $p$ value \\
$\mathrm{n}=1,435$ & $\mathrm{n}=417$ & \\
$(77.5 \%)$ & $(22.5 \%)$ &
\end{tabular}

Migrants who did stay in a migrant shelter $(\mathrm{n}=862)$

Reported some kind of violence

\begin{tabular}{lll}
\hline No & Yes & $p$ value \\
$\mathrm{n}=680$ & $\mathrm{n}=182$ & \\
$(78.8 \%)$ & $(21.1 \%)$ &
\end{tabular}

Sociodemographic characteristics

Gender

Women

73.5

78.7

64.3

28.7

Age (mean in years)

Schooling (mean in years)

Have at least 1 child

Country of origin

Nicaragua

Guatemala

El Salvador

Mexico

Days in transit (mean)

Health problems/accident during this journey $^{\mathrm{a}}$

6.7

24

14.3

64.5

$\begin{array}{rrr}26.5^{*} & 0.023 & 65 . \\ 21.3 & & 80.5 \\ 35.7^{*} & & 42.9 \\ 30.3 & 0.001 & 28 . \\ 6.7 & 0.718 & 6.7 \\ 67.6^{*} & 0.124 & 36.8 \\ & & \\ 4.6 & 0.986 & 7 . \\ 36.2 & & 44 . \\ 21.8 & & 22 . \\ 20.1 & & 24.9 \\ 17.3 & & 1.3 \\ 58.4^{*} & 0.338 & 165 \\ 23.7 * & 0.001 & 18 .\end{array}$

65.5

$34.5^{*}$

19.5

$57.1^{*}$

31.1

7.2

$46.2^{*}$

0.001

63.4

4.9

30.2

26.7

12.8

0.001

7.2

8.2

45.1

22.2

19.8

24.9

24.2

1.3

2.8

$65126^{*}$

$126^{*}$

0.003

$31.3^{*}$

0.001

${ }^{a}$ Included: Injuries, accidents, respiratory disease, gastrointestinal disease, foot fungus, dehydration, etc.

$* p<0.05$ (comparing those who stayed or not in a migrant shelter) 